\title{
Cavern background measurement with the ATLAS RPC system
}

\author{
Giulio Aielli* \\ INFN and University of Roma Tor Vergata \\ E-mail: aiellieroma2.infn.it
}

\section{On behalf of the ATLAS collaboration}

The measurement of cavern background has been carried out systematically since the beginning of LHC, as soon as the luminosity produced a detectable signal on the muon spectrometer, from $\mathscr{L}=10^{28} \mathrm{~cm}^{-2} \mathrm{~s}^{-1}$ of the early 2010 operation up to $\mathscr{L}>3 \times 10^{33} \mathrm{~cm}^{-2} \mathrm{~s}^{-1}$ at the end of 2011 proton-proton run, which is just $1 / 3$ of the nominal LHC luminosity. The reason for this is to early predict the running condition for the detector for the nominal LHC luminosity and beyond, in view of the super-LHC upgrade. Background Montecarlo calculations have been validated against data and the background map analysis pointed out hotspots due to localized cracks in the radiation shielding. The RPCs participated to this effort since the earliest stages providing an accurate correlation between luminosity and background, a 3D background map in the barrel region and a direct measurement of the cavern activation. Moreover, due to the high sensitivity and very good signal to noise ratio of the proposed method, based on the gap current, the measurement was provided in real time through the Detector Control System (DCS), resulting to be a strong diagnostic element of the RPC system. In this presentation the method and the results will be shown in comparison with Montecarlo estimates and other measurements coming from different systems.

XI workshop on Resistive Plate Chambers and Related Detectors - RCP2012,

February 5-10, 2012

INFN Laboratori Nazionali di Frascati Italy

\footnotetext{
* Speaker.
} 


\section{The ATLAS cavern background}

Cavern background refers to the diffuse background from $p-p$ collisions filling the ATLAS detector cavern. It mostly consists of neutral, low energy and long lived particles. Table 1 is the expected background abundance in different areas of the ATLAS muon spectrometer, by particle type and energy. This prediction has been obtained by a FLUKA [1] based simulation for $7 \mathrm{TeV}+$ $7 \mathrm{TeV} p$ - $p$ collisions. The two most abundant particle types are photons and neutrons.

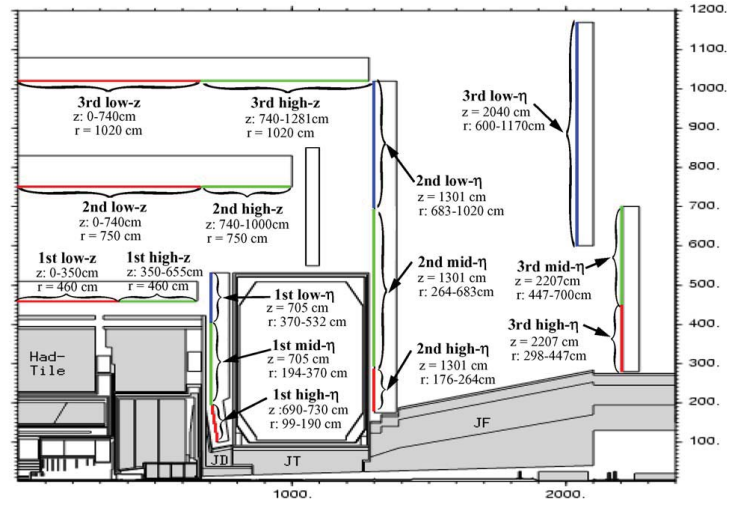

\begin{tabular}{|c|c|c|c|c|c|c|c|c|}
\hline \multirow[b]{2}{*}{ Position } & \multicolumn{4}{|c|}{ Fluences $\left(\mathrm{kHz} / \mathrm{cm}^{2}\right)$} & \multicolumn{4}{|c|}{ Currents $\left(\mathrm{Hz} / \mathrm{cm}^{2}\right)$} \\
\hline & & \multicolumn{2}{|c|}{ Neutrons } & $\begin{array}{c}\text { Photons } \\
>30 \mathrm{keV}\end{array}$ & $\begin{array}{l}\text { Ch.hads } \\
>10 \mathrm{MeV}\end{array}$ & $\begin{array}{c}\text { Protons } \\
>10 \mathrm{MeV}\end{array}$ & $\begin{array}{c}\mu^{+-} \\
>10 \mathrm{MeV} \\
\end{array}$ & $\begin{array}{c}\mathrm{e}^{+-} \\
>05 \mathrm{MeV}\end{array}$ \\
\hline$\frac{\text { Barrel }}{1 \text { 1stlow }}$ & & & & & & & & \\
\hline $\begin{array}{l}\text { 1st,low-z } \\
\text { 1st,high-z }\end{array}$ & $\begin{array}{l}5.08 \\
5.25\end{array}$ & $\begin{array}{l}1.47 \\
1.47\end{array}$ & 0.10 & $\begin{array}{l}1.81 \\
1.88\end{array}$ & $\begin{array}{l}2.79 \\
2.24\end{array}$ & $\begin{array}{l}1.89 \\
186\end{array}$ & $\begin{array}{l}1.89 \\
2.55\end{array}$ & $\begin{array}{r}9.30 \\
13.29\end{array}$ \\
\hline $2 n d, l o w-z$ & 4.97 & 1.20 & 0.10 & 2.19 & 0.69 & 0.64 & 0.37 & 7.00 \\
\hline 2nd, high-z & 6.28 & 1.72 & 0.30 & 4.14 & 1.88 & 1.81 & 0.77 & 11.81 \\
\hline 3rd, low-z & 4.45 & 0.86 & 0.08 & $\begin{array}{l}1.97 \\
1.90\end{array}$ & 0.32 & 0.31 & 0.09 & 5.96 \\
\hline 3rd,high-z & 4.87 & 1.02 & 0.18 & 2.71 & 0.98 & 0.97 & 0.39 & 8.10 \\
\hline$\frac{\text { Forward }}{1 \text { 1stlow-eta }}$ & 14.23 & 480 & 0.66 & 4.93 & 489 & 4.38 & 3.60 & 33.57 \\
\hline 1st,mideta & $\begin{array}{l}14.29 \\
39.33 \\
\end{array}$ & $\begin{array}{l}4.00 \\
14.38\end{array}$ & & & & & & \\
\hline $\begin{array}{l}\text { Ist,mideta } \\
\text { 1st,high-eta }\end{array}$ & $\begin{array}{l}39.33 \\
81.72\end{array}$ & $\begin{array}{l}14.38 \\
34.23\end{array}$ & $\begin{array}{l}1.03 \\
5.72\end{array}$ & $\begin{array}{l}11.64 \\
17.51\end{array}$ & $\begin{array}{l}9.83 \\
40.46\end{array}$ & $\begin{array}{c}8.5 \\
35.1 \\
.\end{array}$ & $\begin{array}{l}13.138 \\
91.38\end{array}$ & $\begin{array}{l}04.90 \\
162.67\end{array}$ \\
\hline $\begin{array}{l}\text { Ist,ingnet } \\
\text { 2nd,low-e }\end{array}$ & $\begin{array}{l}81.72 \\
5.55\end{array}$ & $\begin{array}{l}34.23 \\
1.51\end{array}$ & $\begin{array}{l}5.72 \\
0.25\end{array}$ & ${ }_{3.18}^{17.51}$ & $\begin{array}{l}40.46 \\
1.98\end{array}$ & $\begin{array}{l}35.9 \\
1.9\end{array}$ &  & $\begin{array}{l}12.67 \\
11.91\end{array}$ \\
\hline $\begin{array}{l}\text { 2nd, } \\
\text { 2nd,mid-t }\end{array}$ & $\begin{array}{l}5.53 \\
8.36\end{array}$ & 2.6 .5 & 0.4 & & & 6.2 .5 & 1. & $\begin{array}{l}\begin{array}{l}11.91 \\
23.58\end{array} \\
\end{array}$ \\
\hline $\begin{array}{l}\text { 2nd, mideta } \\
\text { 2nd, high-eta }\end{array}$ & $\begin{array}{r}8.36 \\
16.70\end{array}$ & $\begin{array}{l}2.6 \\
5.8\end{array}$ & $\begin{array}{l}.49 \\
1.23\end{array}$ & 8 & $\begin{array}{l}6.45 \\
19.55\end{array}$ & $\begin{array}{l}6.20 \\
17.63\end{array}$ & 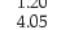 & $\begin{array}{l}23.58 \\
57.90\end{array}$ \\
\hline & 3.16 & 0.48 & 0.06 & & 1.53 & 1.4 & 0.2 & 7.41 \\
\hline & $\begin{array}{l}3.16 \\
3.09\end{array}$ & 0.48 & $\begin{array}{l}0.06 \\
0.05\end{array}$ & $\begin{array}{l}1.4 \\
1.4 \\
\text {. }\end{array}$ & $\begin{array}{l}1.5 \\
1.2\end{array}$ & 1.4 & $\begin{array}{l}0.27 \\
0.32\end{array}$ & $\begin{array}{l}7.41 \\
6.41\end{array}$ \\
\hline $3 \mathrm{rdh}$ & 2.05 & 0.48 & 0.05 & 1.21 & $\begin{array}{l}1.25 \\
1.06\end{array}$ & $\begin{array}{l}1.21 \\
0.97\end{array}$ & 0.46 & $\begin{array}{l}0.41 \\
7.32\end{array}$ \\
\hline
\end{tabular}

Figure 1: Typical distribution of background by particle type and energy in the muon spectrometer region predicted in a FLUKA-based simulation.

The cavern background study is necessary to quantify the background levels and how they may evolve with the LHC machine luminosity and energy and to verify the Montecarlo prediction produced before the LHC start with very large uncertainties. This is crucial to check the present detector reliability and to design in advance the upgrades for detectors and shielding having the appropriate performance.



Figure 2: Views of the ATLAS muon system: y-z plane (top) and x-y plane (bottom).

The cavern background is actually measured by the Muon system [2], shown in Figure 2, to guarantee the maximum possible coverage in terms of detector volume and to compare the different 
detector technology response to the background exposure, in terms of sensitivity and measurement systematic errors. In the barrel region $(|\eta|<1.05)$ the comparison is ensured by the MDTs and RPCs detectors assembled in three concentric layers called inner (BI), middle (BM) and outer (BO). RPCs and MDTs are bundled in to muon stations everywhere (except in the innermost region) thus they can be easily compared for the background measurement. The barrel is characterized by a lower background intensity with respect to the forward region (endcap), largely dominated by photon hits, and negligible beam halo contamination, allowing very clean measurements. The RPC middle layer is made of two chambers mounted on the innermost and outermost side of the MDTs, called Confirm and Pivot planes respectively. The chambers are organized in 16 sectors along the azimuthal coordinate $(\Phi)$, to facilitate the overlap the even sectors are slightly smaller than the odd ones thus we refer respectively to large (L) and small (S) chambers. Two principal measurement strategies have been pursued, complementary to each other, based respectively on hits collected through the DAQ and detector currents collected through DCS.

In the first case hits from all the muon chambers are read out via the event data acquisition path. To minimize the bias from the trigger the data come from minimum bias or random filled trigger stream. The result is sensitive to the pileup effect due to the bunch structure of the beam and to the long tail of the time spectrum of the hits correlated to the bunch crossing

The HV current measurements integrate the signal much longer than the LHC revolution frequency therefore are insensitive to the bunch structure and to the trigger. Their spatial granularity is generally poorer than that from hits which is driven from the chamber granularity. In general the charge signal amplitude depends on the primary ionization which depends on the type of particles. This uncertainty has to be taken in to account through appropriate conversion factors.

\section{The RPC gap current based method}

The detection of a ionizing particle is associated to the production of an electron avalanche in the gas. The total charge delivered has to be compensated by the power supply with a time constant of about $20 \mathrm{~ms}$ driven by the total capacitance and resistance of the system [3]. The average value of the detector current, cleaned up from all detector systematic contributions, represents the average charge per second delivered due to the incident particle rate. By knowing the average charge per particle delivered in the gas $\left(<Q_{t o t}>\right.$ and the gap current $I_{g a p}$ and its area $A_{\text {gap }}$, is then possible to calculate the average particle rate $\mathrm{R}$ incident on each gap:

$$
R=\frac{I_{g a p}}{<Q_{t o t}>A_{g a p}}
$$

The sensitivity is driven by the precision of the volt-amperometric method used to measure the detector current flowing on a $100 \mathrm{k} \Omega$ resistor in parallel to a $10 \mathrm{nF}$ capacitor, placed in series on the HV return wire before joining with the common detector ground. The voltage drop is read across the resistor with CAEN A1801 a 128 channels ADC module having a sensitivity of $0.2 \mathrm{mV}$ which corresponds to $2 \mathrm{nA}$, equivalent to about $30 \mathrm{~Hz} / \mathrm{m}^{2}$ of particle rate. The instrument can average a programmable number of $1 \mathrm{~ms}$ samples up to 250 , within a readout cycle of about $1 \mathrm{~s}$. Thus the measurement duty cycle is very high if compared to the hit counting based method of about $4 \times 10^{-5}$ given by the readout window ( $2 \mu$ s for the MDTs and 200ns for the RPCs) multiplied 
by the data acquisition rate of the order of $200 \mathrm{~Hz}$. By this means the time necessary to collect an equivalent sample is about 6000 times faster, allowing in principle sensitive measurement at very low luminosity [4] $\left(\mathscr{L}=6 \times 10^{28} \mathrm{~cm}^{-2} \mathrm{~s}^{-1}\right.$ is the lowest measured signal). To enhance the statistics the DCS acquire and average several current measurements, recording the resulting moving average about every minute [5]. Moreover the working point correction performed by the DCS remove the environmental effects.

The 3592 RPC gas gaps installed in the ATLAS barrel region are organized in doublets of identical gaps. A chamber can contain up to 4 doublets coupled side by side in $\Phi$ or in $\mathrm{Z}$ direction, or both [5]. The statistical combination of all the gaps provides the best possible estimate of the average background rate in the RPC area. A conversion factor of $30 \mathrm{pC} /$ count at the standard working point of $9600 \mathrm{~V}\left(\mathrm{P}=975 \mathrm{mbar}\right.$ and $\mathrm{T}=24^{\circ} \mathrm{C}$ been estimated for photons at the CERN GIF (Gamma Irradiation Facility) with a ${ }^{137} \mathrm{Cs}$ source $[6,7]$ and with a ${ }^{60} \mathrm{Co}$ source in laboratory tests [8]. The conversion factor for MIPS is about 20pC. Other cases such as neutrons, are neglected in this analysis. This approximation is justified by the fact that on one hand in the barrel the neutron hits are negligible with respect to the photons due to the very low sensitivity [?]; on the other hand an highly saturated regime should strongly suppress the signal charge dynamical range [9].

\subsection{The off-line current analysis}

The raw rata from the RPC currents are reprocessed off-line to improve the quality of the measurement cleaning up the noisy channels and improving the statistical error. To cleanup the noise the facing gaps of the same doublet (the minimal RPC subset measuring the background in 3D) are compared. Having the same surface and position are very correlated to the background intensity. The average RPC working current without beam is of the order of $100 \mathrm{nA} \mathrm{m} \mathrm{m}^{-2}$ and it is subtracted using the calibration data after each beam dump. The best value for the doublet is determined looking at fig. 3. If the difference of the two currents is less than $20 \%$ the average current will be taken as representative of the doublet; otherwise the lowest of the two is taken. Other checks are applied to avoid fake values due to disconnected gaps or readout failures or known noisy cases. The rate in $\mathrm{Hz} \mathrm{cm}^{2}$ is obtained by using $30 \mathrm{pC} /$ count as explained above.

The map shown in fig. 3 represents the rate values as a function of doublet position within the ATLAS barrel in cylindrical coordinates. The radius corresponds to the chamber layer distance from the beam axis (e.g. middle and outer layer stations); the longitudinal coordinate $\mathrm{Z}$ is given by the RPC unit identifier along the beam axis (values are given relatively to the interaction point considering the clockwise circulating beam, named beam 1); the azimuthal coordinate $\Phi$ is given by the sector and semi sector identifiers running clockwise around beam 1 . These maps show counting rate in $\mathrm{Hz} \mathrm{cm}^{2}$ in each bin, the color scale, given to help the plot understanding is generated by a series of proportional shades of color normalized to the maximum and minimum rate values. The $\mathrm{Z}$ and $\Phi$ projective averages are also provided, as well as the global average at the bottom right corner of each table. In general we observe that the rate tend to increase at higher $\mathrm{Z}$ for the same radius as expected due to the higher value of $\eta$; moreover the systematic rate difference between odd and even sectors due to the effect of the toroid coils shadowing the even sectors and acting as absorber material. Going more in details two hot regions are visible, in particular in the innermost chamber layer for chamber id along $\mathrm{Z}= \pm 5$. This is due to the known unshielded crack present until 2011 between the calorimeter and the small wheel shielding, called J-disk as described in [2]. For 

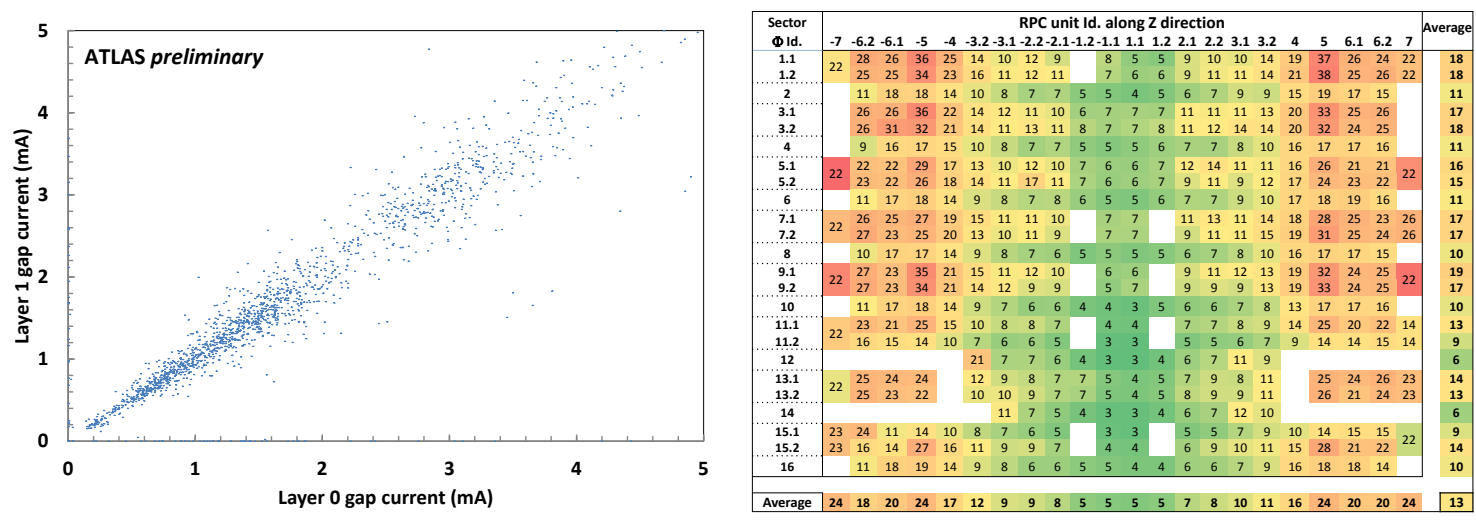

Figure 3: (Left) Scatter plot of measured current of homologous gas gaps occupying the same position in the space while belonging to different layers. (Right) Average background rate distribution map for the RPC middle for $\mathscr{L}=10^{34} \mathrm{~cm}^{-2} \mathrm{~s}^{-1}$. The rate in $\mathrm{Hz} \mathrm{cm}^{2}$ is shown for each individual gap position identified by its longitudinal and azimuthal position

the 2012 runs the shielding has been improved to suppress the rate excess. The feet region in the lowest part of the barrel (sectors 12,13 and 14) shows a lower counting rate due to the presence of the ATLAS feet steel supports.

\section{Data - Monte Carlo Comparisons}

A FLUGG-based application simulates individual proton-proton collisions whose daughter particles are followed through the ATLAS detector materials. As they enter the muon detectors logical volumes they are scored and converted to particle fluxes according to the respective sensitivity functions which depend on the particle type and energy. More details about the FLUGG-based simulation can be found in [?].

The comparison of RPC data vs. FLUGG predictions is performed by converting the MC counts in each scoring volume to an equivalent rate at a given luminosity using the following:

$$
\mathrm{MC} \text { hit rate }\left[\mathrm{Hz} / \mathrm{cm}^{2}\right] R_{\mathrm{MC}}=\frac{n_{\mathrm{hit}, \mathrm{MC}}}{\varepsilon n_{\mathrm{events}, \mathrm{MC}}} \cdot \mathscr{L} \cdot \sigma_{\mathrm{pp}} \cdot \frac{1}{A}
$$

, $\mathscr{L}$ corresponds to the instantaneous luminosity at which the data have been sampled $n_{\text {hit,MC }}$ the number of hits on a given surface $A, n_{\text {events,MC }}$ the total number of simulated events, $\varepsilon$ an enhancement factor for the detector sensitivity to improve the statistics, and $\sigma_{\mathrm{pp}}$ the proton-proton collision cross section.

In the present case, the LHC fill 2110 starting 15/9/2011 has been taken as a reference and the data represent the average of three consecutive sample of $30 \mathrm{~s}$ each with an average luminosity of $2.94 \times 10^{33} \mathrm{~cm}^{-2} \mathrm{~s}^{-1}$. To improve the comparison significance and to overcome the statistics limitation of the present FLUGG sample the data and the Montecarlo hits have been summed over respectively the coordinate $\Phi$ and $\mathrm{Z}$ of the barrel, obtaining $\mathrm{Z}$ and $\Phi$ projections. The comparison study is summarized in figures 4 and 5 where are shown the $\mathrm{Z}$ projection of the odd and even sectors and the $\Phi$ projection of all sectors respectively. Here the data are normalized to $\mathscr{L}=$ $10^{33} \mathrm{~cm}^{-2} \mathrm{~s}^{-1}$. A bin on these plots thus represents an area corresponding to the gas gap extension 
on the coordinates axis, integrated on the other coordinate over the ATLAS barrel. The data are further integrated on the two gas gap layers of a chamber, and for the middle stations the Pivot and Confirm chambers have been summed together as in the FLUGG simulation they correspond to a single scoring volume. Due to minor mismatches between the scoring volumes on the MC simulation and the real chamber sizes in some specific detector area the MC counts have been scaled linearly to fit the real chamber areas. Each projection is completed with an auxiliary plot showing per each bin the fractional difference between data and MC: $($ Data $-M C) /$ Data. Each figure shows from left to right the middle layer and the outer layer chambers results respectively.
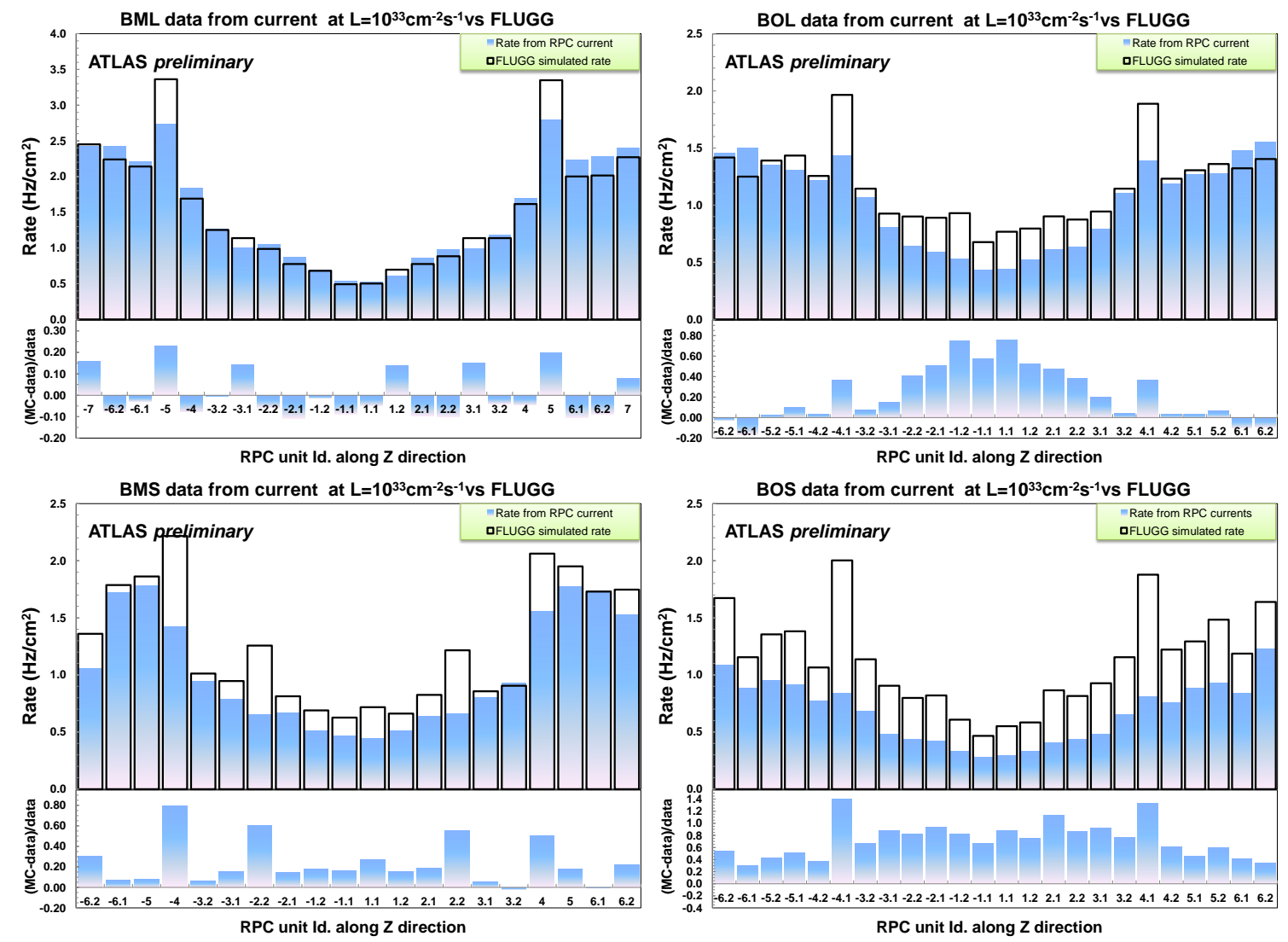

Figure 4: Data to FLUGG-based simulation comparison for large (even) and small (odd) sectors of the middle and outer layers. The data are integrated over the azimuthal coordinate $\Phi$ and projected on the $Z$ axis. Each bin of the top panel shows the rate in $\mathrm{Hz} \mathrm{cm}^{-2}$ averaged on all the gas gaps with the same position in $\mathrm{Z}$, indicated by the chamber id.

As noted before there are localized excess of MC counts with respect to the measured signal in correspondence of the shielding cracks at $z \sim 3 \mathrm{~m}$ and $z \sim 7 \mathrm{~m}$. Another systematic effect is visible on the outer layer chambers showing a significative lower counting in the central values of $\mathrm{Z}$ with respect to the MC. This effect is much reduced on the middle layer chambers and almost suppressed on the BMLs. Looking at $\Phi$ projection it is clear that the even sectors show an overall effect of lower counting with respect to the $\mathrm{MC}$ at all $\mathrm{Z}$ values, more pronounced in the outer layer chambers; this overall effect is systematically concentrated in the lowermost sectors.

An analysis of this discrepancies can be done by separating the scaling factor error which 

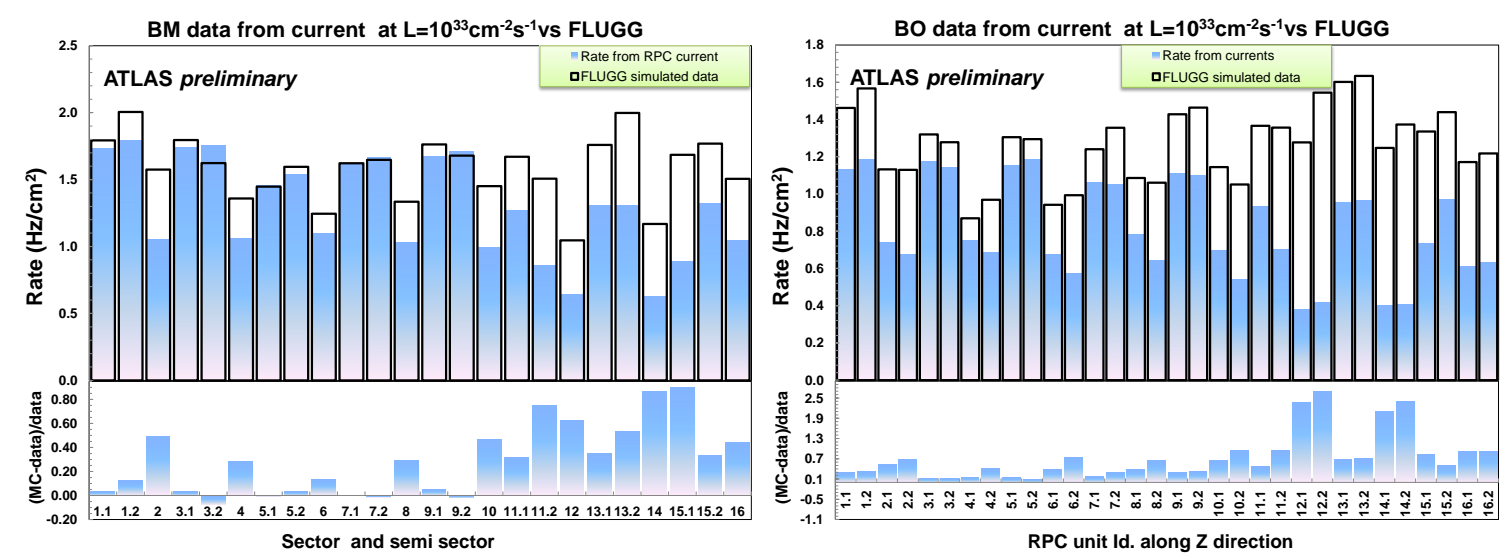

Figure 5: Data to FLUGG-based simulation comparison for the middle and outer layers. The data are integrated over the coordinate $\mathrm{Z}$ and projected on the $\Phi$ axis. Each bin of the top panel shows the rate in $\mathrm{Hz}$ $\mathrm{cm}^{-2}$ averaged on all the gas gaps within the same position in $\Phi$, generally corresponding to an extension of one half of a sector, except for the BMS chambers having only one gap covering the sector width.

may be attributed to a conversion factor and/or to the sensitivity function hardwired in the MC. In both cases we must also consider a non uniform term due to the approximations concerning the heavy ionizing particles such as the neutrons, which have a non uniform distribution. This effect would show as a apparent excess of counts where there is an higher concentration of neutron hits (typically at higher $\eta$ ). A better knowledge of the neutron distribution and detector response function is needed to go beyond the present level of comprehension at this point. The systematic effect on the differences shown in the distributions of figures 4 and 5, seems to be related to the passive material distribution which is more concentrated in the ATLAS barrel feet area.

\section{References}

[1] S. Baranov et al.; "Estimation of Radiation Background, Impact on Detectors, Activation and Shielding" ATL-GEN-2005-001

[2] The ATLAS Collaboration; Cavern background measurements and comparison with simulation; 2012; ATLAS note in preparation

[3] G. Aielli et al.; The RPC current time structure. Fast current peak measurement in the ATLAS RPC system; Nucl. Instr. and Meth. A 661 (2012) 201-205.

[4] M. Bindi; " ATLAS RPC detector as Luminosity monitor"; RPC2012; In this proceedings

[5] A. Polini; "Performance and Operation of the ATLAS RPC Detector during the 2011 LHC run" RPC2012; In this proceedings

[6] G. Aielli et al.; RPCs ageing studies; Proc. of VCI 2001, Nucl. Instr. and Meth. A 478 (2002) 271-276

[7] G.Aielli et al.; "Ageing test of the ATLAS RPCs at X5-GIF"; Nucl. Instr. and Meth. A 533 (2004) 98-101

[8] G. Aielli et al.; An RPC $\gamma$ irradiation test; Nucl. Instr. and Meth. A 414 (1998) 82-86.

[9] G. Aielli et al.; "Saturated logistic avalanche model"; B Ě - Nucl. Instr. and Meth. A 508 (2003) 6-13 